\title{
Relative Adrenocortical Insufficiency Following Radioactive lodine Therapy for Graves' Disease: A Report of Two Cases
}

\author{
Hanxin Zhao \\ Yu Ruan \\ Department of Endocrinology and \\ Metabolism, Zhejiang University Affiliated \\ Sir Run Run Shaw Hospital, School of \\ Medicine, Hangzhou, People's Republic of \\ China
}

Introduction: In recent years, radioactive iodine (RAI) therapy has become a main choice for Graves' disease. The rapid release of thyroid hormones following RAI may on occasion trigger severe events, such as thyroid storm or heart block. This study presents two cases of possible acute adrenocortical insufficiency precipitated by radioiodine therapy.

Case Presentation: Two females aged 65 and 19 years with long-standing Graves' disease underwent radioiodine treatment at our clinical center. Both patients suffered nausea, fatigue, aggravated palpitation, and relative hypotension thereafter. Laboratory data showed severe thyrotoxicosis with free thyroxine higher than three times the upper limit, while basal serum cortisol ( $8 \mathrm{AM})$ was below the lower limit $(5.08 \mu \mathrm{g} / \mathrm{dL}$ and $2.08 \mu \mathrm{g} / \mathrm{dL}$ respectively) under stress, indicating a potential relative adrenocortical insufficiency. There was then full recovery after adequate hydrocortisone therapy. As thyrotoxicosis resolved, the levels of basal cortisol were subsequently raised to normal.

Conclusion: Post-RAI adrenocortical insufficiency might occur in patients with severe Graves' disease. Basal serum cortisol might be a cost-effective parameter for the identification of potential patients.

Keywords: Graves' disease, hyperthyroidism, adrenal insufficiency, radioiodine therapy

\section{Introduction}

China is a populous country with a high incidence of Graves' hyperthyroidism $(1.1 \%-1.4 \%){ }^{1}$ Nowadays, an increasing number of Chinese patients come to accept radioactive iodine (RAI) therapy, ${ }^{2}$ which has been considered efficient and well tolerated for decades. ${ }^{3}$ As guidelines advised, ${ }^{3}$ physicians should fully communicate with patients about the benefits, expected speed of recovery, drawbacks, and potential side effects. Therefore, any unusual emergency that occurs at any point in time during medical practice deserve attention.

Previous studies have shown that thyroxine could enhance the clearance and synthetic rate of cortisol, with a balanced net effect most of the time. ${ }^{4,5}$ When people are in stress like rapidly worsened hyperthyroidism, or new onset thyrotoxicosis in patients with primary adrenocortical insufficiency, a latent adrenal crisis may emerge. Due to the low incidence and confusing manifestations, diagnosis might be delayed. ${ }^{6-8}$

In this study, to highlight the masked threatening condition, we describe two cases of Graves' disease who developed relative adrenocortical insufficiency post-RAI therapy. 


\section{Case Presentation}

\section{Case I}

A 65-year-old woman with palpitation and weight loss presented to our hospital for RAI therapy. She was diagnosed to have Graves' disease one month previously. After taking methimazole (MMI) $10 \mathrm{mg}$ for three weeks, she was found to have mild leukopenia (white-cell count 2940/ $\mathrm{mm}^{3}, 1730$ neutrophils $/ \mathrm{mm}^{3}$ ). She reported no fever, cough, diarrhea, stomachache, nausea, vomiting, or dizziness. The patient had received montelukast and cetirizine hydrochloride for chronic bronchitis. Her heart rate was 98 bpm and blood pressure 136/79 mmHg. On physical examination, the goiter was enlarged with no tenderness. The lung was clear and heart rate was regular, without a murmur. She was found to have an undetectable thyroid stimulating hormone (TSH) of $0.00 \mathrm{mIU} / \mathrm{L}(0.35-4.94$ $\mathrm{mIU} / \mathrm{L}$ ) and an elevated free thyroxine (FT4) level of $1.62 \mathrm{ng} / \mathrm{dL}(0.7-1.48 \mathrm{ng} / \mathrm{dL})$. Other parameters are shown in Table 1. An electrocardiogram (ECG) revealed sinus rhythm, a ventricular rate of $96 \mathrm{bpm}$. Echocardiography revealed normal left ventricular systolic function with an ejection fraction of $66 \%$. A technetium- $99 \mathrm{~m}$ thyroid scintigraphy revealed diffusely elevated uptake in the goiter and a thyroid weight of $81.97 \mathrm{~g}$. RAI uptake test demonstrated $62.8 \%$ uptake in $24 \mathrm{~h}$. Due to her concern over the side effects of anti-thyroid drugs (ATD), the patient chose RAI treatment. After the correction of leucopenia, she received ${ }^{131} \mathrm{I}$ administration of $10 \mathrm{mCi}$ in March 2017, and was discharged on metoprolol.

Two weeks later, the patient returned to the emergency department presenting with appetite loss, nausea, vomiting, fatigue, edema, and aggravated palpitation. Upon arrival, she had sinus tachycardia (113 bpm), relative hypotension (92/ $60 \mathrm{mmHg}$ ), with a normal temperature. Physical examination revealed emaciation, dysphoria, diaphoresis, diffuse non-tender thyromegaly (Grade II), and mild pitting edema of the eyelids and lower extremities. The lung was clear, her abdomen was not tender, and murphy's sign was negative. Thyroid function showed exacerbated thyrotoxicosis: TSH $0.00 \mathrm{mIU} / \mathrm{L}$, FT4 $4.48 \mathrm{ng} / \mathrm{dL}$, free triiodothyronine (FT3) $>30.00 \mathrm{pg} / \mathrm{mL}(1.71-3.71 \mathrm{pg} / \mathrm{mL})$, thyrotrophin receptor antibody (TR-Ab) $37.47 \mathrm{IU} / \mathrm{L}(0.00-1.22 \mathrm{IU} / \mathrm{L})$. The whitecell count was $4500 / \mathrm{mm}^{3}$, and neutrophils $2800 / \mathrm{mm}^{3}$. The sodium level was $137 \mathrm{mmol} / \mathrm{L}$, potassium level $4.0 \mathrm{mmol} / \mathrm{L}$, chloride level $104 \mathrm{mmol} / \mathrm{L}$, and fasting blood glucose was $4.77 \mathrm{mmol} / \mathrm{L}$. The levels of plasma troponin, NT-proBNP, serum hepatic enzymes, bilirubin, and creatinine were all within the reference range.

Given that the patient developed symptoms in a short time after radioactive therapy, thyroid storm was the first probable diagnosis. However, she had no fever, no signs of central nervous system disturbance, infection, or heart failure. According to the Burch-Wartofsky Point Scale or Japanese Thyroid Association criteria, ${ }^{9}$ neither early stage or suspected thyroid storm were supported. Given her weakness, appetite loss, gastrointestinal symptoms, hypotension, and low-normal serum sodium, adrenocortical insufficiency had to be excluded. Laboratory data revealed decreased basal serum cortisol $(5.08 \mu \mathrm{g} / \mathrm{dL})$ and elevated plasma adrenocorticotropic hormone (ACTH) $(112 \mathrm{ng} / \mathrm{L})$ under such stress. Her sexual hormone profile revealed a normal post-menopausal state. Abdominal and adrenal

Table I Thyroid Function Profile and Serum Cortisol Level in Case I

\begin{tabular}{|c|c|c|c|c|c|c|}
\hline Time After RAI & Day of RAI & 2 Weeks & 2 Months & 3 Months & 6 Months & Reference \\
\hline TSH (mIU/L) & 0 & 0 & 0 & 0 & 0 & $0.35-4.94$ \\
\hline TT3 (ng/mL) & 1.76 & $>8.00$ & 2.42 & 2.04 & 1.48 & $0.58-1.59$ \\
\hline TT4 ( $\mu g / d L)$ & 9.9 & 21.05 & 12.87 & 6.79 & 5.05 & $4.87-11.72$ \\
\hline FT3 (pg/mL) & 5.95 & $>30.0$ & NA & 6.76 & 4.31 & $|.7|-3.7 \mid$ \\
\hline FT4 (ng/dL) & 1.62 & 4.48 & NA & 1.36 & 0.86 & $0.7-1.48$ \\
\hline TR-Ab (IU/L) & 6.33 & 37.47 & $>40.0$ & $>40.0$ & $>40.0$ & $0.00-1.22$ \\
\hline $\begin{array}{l}\text { Basal Cortisol } \\
\text { (8AM, } \mu g / d L)\end{array}$ & NA & 5.08 & 9.95 & 8.08 & 10.5 & $6.70-22.6$ \\
\hline
\end{tabular}

Note: Thyroid function profiles were measured by chemiluminescence microparticle immuno assay and serum cortisol was measured by chemiluminescence immunoassay. Abbreviations: TSH, thyroid stimulating hormone; TT3, total triiodothyronine; TT4, total thyroxine; FT3, free triiodothyronine; FT4, free thyronine; TR-Ab, thyrotrophin receptor antibody; NA, not available. 
ultrasound findings were unremarkable. Since the patient had no history or presentation of pituitary or adrenal disorders, and she had no history of exposure to exogenous glucocorticoid, a diagnosis of acute adrenocortical insufficiency following RAI therapy was highly suspected.

Subsequently, $100 \mathrm{mg}$ hydrocortisone succinate was given intravenously followed by appropriate fluid resuscitation. Her symptoms diminished soon and her blood pressure was normalized (130/74 $\mathrm{mmHg})$. She was then placed on tapered hydrocortisone and propylthiouracil (PTU). On the day of discharge, she was prescribed $20 \mathrm{mg}$ hydrocortisone and $100 \mathrm{mg}$ PTU daily. At two months follow-up, the patient stated that she had no discomfort and discontinued hydrocortisone by herself one week prior. Labs at that time showed normalized levels of basal cortical $(9.95 \mu \mathrm{g} / \mathrm{dL})$ and serum electrolytes (sodium level $142 \mathrm{mmol} / \mathrm{L}$, potassium level $4.1 \mathrm{mmol} / \mathrm{L}$ ). PTU was then reduced to $50 \mathrm{mg}$ daily based on thyroid function. It remained stable during the subsequent follow-up visits. The relation of her FT4, FT3, and cortisol levels over time is demonstrated graphically in Figure 1.

\section{Case 2}

A 19-year-old female was admitted to our department for RAI therapy in July 2018 due to her 10-year recurrent Graves' hyperthyroidism. She had a parental family history of Graves' disease. She was on $10 \mathrm{mg}$ propranolol three times a day and MMI $10 \mathrm{mg}$ once daily. Her heart rate was $113 \mathrm{bpm}$ and blood pressure was $124 / 74 \mathrm{mmHg}$. On physical examination, the goiter was visibly enlarged with a murmur, no edema, pigmentation or hair loss. Her heart rate was regular, and an ECG revealed sinus tachycardia $(110 \mathrm{bpm})$ and a prolonged corrected QT interval. Blood tests indicated severe hyperthyroidism (TSH 0.00 $\mathrm{mIU} / \mathrm{L}$; FT4 $4.41 \mathrm{ng} / \mathrm{dL}$; FT3 $>30.00 \mathrm{pg} / \mathrm{mL}$; TR-Ab $>40.0$ $\mathrm{IU} / \mathrm{L})$. A technetium-99m thyroid scintigraphy revealed

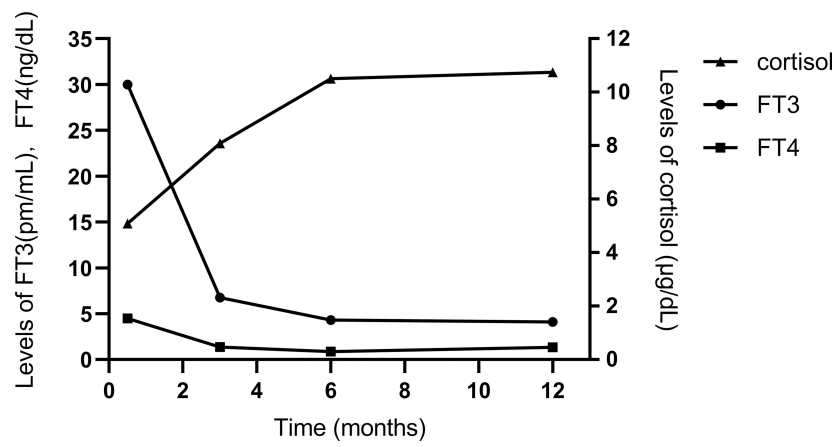

Figure I Graphic demonstration of FT4, FT3, and cortisol levels over time in case I. diffusely elevated uptake in the goiter and a thyroid weight of $210.04 \mathrm{~g}$. RAI uptake test showed a homogenous gland with $69.4 \%$ uptake in $2 \mathrm{~h}$ and $65.5 \%$ in $24 \mathrm{~h}$. MMI was discontinued three days prior to ${ }^{131} \mathrm{I}$ administration. A dose of $12 \mathrm{mCi}$ radioiodine was administered orally and propranolol was prescribed $10 \mathrm{mg}$ every six hours.

Three days after ${ }^{131}$ I administration, she developed appetite loss, vomiting, fatigue, headache, and aggravated palpitation. When she presented to our hospital the day after symptom onset, her temperature was $36.9^{\circ} \mathrm{C}$, heart rate was $122 \mathrm{bpm}$ and blood pressure was 105/65 $\mathrm{mmHg}$. On physical examination, the goiter was visibly enlarged and non-tender. Abdominal and neurological examinations were unremarkable. The levels of FT4 and FT3 were higher than the upper limit (details in Table 2). The sodium level was $133 \mathrm{mmol} / \mathrm{L}$, potassium level 4.33 $\mathrm{mmol} / \mathrm{L}$, and chloride level $102 \mathrm{mmol} / \mathrm{L}$. The highsensitivity C-reactive protein, erythrocyte sedimentation rate, hepatic enzyme spectrum, and renal function were all within normal range. Random serum cortisol was 2.08 $\mu \mathrm{g} / \mathrm{dL}$ under such stress. Craniocerebral and abdominal CT scan was unrevealing.

Similarly, combined with her history and testing results, a probable diagnosis of acute adrenocortical insufficiency was considered. In the emergency room, $50 \mathrm{mg}$ hydrocortisone succinate was given intravenously, which provided immediate relief. She was discharged later on hydrocortisone orally for three days.

One month later, she returned to the clinic without discomfort. Her basal serum cortisol level was raised to normal (cortisol $11.14 \mu \mathrm{g} / \mathrm{dL}$ ). The levels of FT4, TT4, FT3, and TT3 were still higher than the upper limit. She resumed taking MMI $20 \mathrm{mg}$ daily and the dose varied depending on thyroid function. One year later, the patient received a second dose of ${ }^{131} \mathrm{I}$ administration $(10 \mathrm{mCi})$ under close observation. This time, she reported no fever, no appetite loss, no nausea, or headache and had a normal level of basal serum cortisol $(8.79 \mu \mathrm{g} / \mathrm{dL})$ after RAI therapy.

\section{Discussion}

The cooperative effects of thyroxin and glucocorticoids have been well documented. In clinical practice, adrenocortical insufficiency, a potentially life-threatening syndrome may occur when thyrotoxicosis is exacerbated. It has been reported in situations such as misuse of thyroid hormone in a background of latent adrenal insufficiency, ${ }^{10,11}$ new onset thyrotoxicosis in a patient with congenital adrenal 
Table 2 Changes of Thyroid Function and Serum Cortisol in Case 2

\begin{tabular}{|l|c|c|c|c|c|c|c|}
\hline Time After RAI & Day of RAI & 3 Days & I Month & I 2 Month* & I4 Months & 28 Months & Reference \\
\hline TSH $(\mathrm{mlU} / \mathrm{L})$ & 0 & 0 & 0 & 0 & 0.59 & 0 & $0.35-4.94$ \\
\hline TT3 $(\mathrm{ng} / \mathrm{mL})$ & $>8.00$ & $>8.00$ & $>8.00$ & $>8.00$ & 0.39 & 1.15 & $0.58-1.59$ \\
\hline TT4 $(\mu \mathrm{g} / \mathrm{dL})$ & 22.84 & $>24.0$ & $>24.0$ & $>24.0$ & 2.06 & 7.52 & $4.87-I I .72$ \\
\hline FT3 $(\mathrm{pg} / \mathrm{mL})$ & $>30.0$ & $>30.0$ & $>30.0$ & $>30.0$ & 1.17 & 3.66 & $1.71-3.71$ \\
\hline FT4 $(\mathrm{ng} / \mathrm{dL})$ & $4.4 I$ & $>5.0$ & $>5.0$ & 4.7 & 0.47 & 1.22 & $0.7-1.48$ \\
\hline TR-Ab $(\mathrm{IU} / \mathrm{L})$ & $>40.0$ & $>40.0$ & $>40.0$ & $>40.0$ & $>40.0$ & 38.96 & $0.00-1.22$ \\
\hline $\begin{array}{l}\text { Basal Cortisol } \\
(8 A M, \mu g / d L)\end{array}$ & NA & 2.08 & 11.14 & 8.79 & NA & NA & $6.70-22.6$ \\
\hline
\end{tabular}

Note: *The day around the second ${ }^{|3|} \mathrm{I}$ administration.

Abbreviations: TSH, thyroid stimulating hormone; TT3, total triiodothyronine; TT4, total thyroxine; FT3, free triiodothyronine; FT4, free thyronine; TR-Ab, thyrotrophin receptor antibody; NA, not available.

hyperplasia, ${ }^{6}$ or secondary adrenal insufficiency, ${ }^{8}$ and simultaneous presentation of Graves' disease and Addison's disease. $^{7}$

Previous research demonstrated the association between long-standing thyrotoxicosis and deficient adrenocortical reserves, ${ }^{12-16}$ despite the lasting hyperactivity of the hypothalamic-pituitary-adrenal axis. ${ }^{17}$ In druginduced hyperthyroid rat models, plasma corticosterone could be effectively increased in response to ACTH release. ${ }^{15}$ Similarly, in humans, due to the elevated metabolic rates, severe thyrotoxicosis may lead to adrenocortical insufficiency, which presents as significant hypocortisolemia or inadequate cortisol response to ACTH stimulation. ${ }^{12-14}$ Although it often occurs in a subclinical state and would resolve after the attainment of euthyroidism, patients are at increased risks of adrenal crisis $^{10,17,18}$ when in stress of diarrhea, infection, surgery, or experiencing a sharp release of thyroid hormones. Conversely, evidence has shown that Graves' disease shares a similar process with other autoimmune endocrine diseases like autoimmune thyroiditis, thyroid eye disease, Type 1 diabetes and Addison's disease through cytokines. ${ }^{19,20}$ Hence, adrenal insufficiency might be a result of immune response to Graves' thyrotoxicosis under certain stimuli.

For Graves' thyrotoxicosis, RAI treatment, ATD, and thyroidectomy are main modality therapies. Despite the regional preferences, RAI has become more popular given its safety and efficiency. ${ }^{21}$ According to the American thyroid Association Guideline for Hyperthyroidism in 2006, ${ }^{3}$
$59.7 \%$ of respondent clinical endocrinologists from the United States selected RAI as primary choice, compared to $32 \%$ among Chinese physicians. ${ }^{2}$ During previous clinical practice, much attention has been given to radiation thyroiditis, which is caused by a transient exacerbation of hyperthyroidism. ${ }^{22}$ Actually, factors like inaccurate dose due to potential changes of pre- to intra-therapeutically biokinetics of ${ }^{131}$ I may induce side effects such as thyroid swelling, sialadenitis, or immune response. ${ }^{23-25}$ RAI therapy may even trigger rare complications like thyroid storm and heart block. ${ }^{26}$

Combined with the aforementioned points, one may reasonably conceive that some patients would suffer symptoms of cortisol deficiency in the post-RAI period due to the short-term worsening of hyperthyroidism or the possible immune interaction. As far as is known, there is yet no such report of a clinical study or observation.

In the current study, we report two cases with Graves' thyrotoxicosis that manifested similarly with loss of appetite, fatigue, and aggravated palpitation in a short time after radioactive therapy, in whom the diagnosis was presumed to be radiation thyroiditis or the early stage of thyroid storm. However, further tests revealed low AM serum cortisol in the face of stress, indicating a likely underlying acute adrenocortical insufficiency, or even concomitant with thyroid storm. At the initial presentation of case 1, given the test result of high ACTH and hypocortisolemia, we were not sure whether she had prior primary adrenal insufficiency or not. Then through close follow-up, we discovered that 
the patient no longer needed glucocorticoid replacement and achieved normalized cortisol and ACTH as FT3, FT4 trended down, which confirmed our suspicion that this was a transient adrenocortical insufficiency. ${ }^{27}$ On this base, we started to evaluate adrenocortical function for patients with severe thyrotoxicosis after ${ }^{131}$ I administration. Most patients had normal basal cortisol except the second case. The girl had a 10-year course of Graves' disease, with an extremely high level of FT3, FT4, and elevated uptake of technetium$99 \mathrm{~m}$. Within a short time after RAI therapy, she soon exhibited classic presentations of acute adrenocortical insufficiency with hypocortisolism. After timely supplement of hydrocortisone her symptoms diminished rapidly and she finally attained normalized cortisol level as FT3, FT4 went down. However, there were several limitations to the present study that warrant discussion. First, none of the patients accepted adrenal stimulation testing during convalescence due to personal reasons. An entire evaluation would provide results that are more reliable. Second, cortisol-binding globin was not available in our center, which was a possible confounder as it might be low in patients with thyrotoxicosis. ${ }^{16}$ Third, the intra-therapeutic ${ }^{131}$ I uptake and effective half-life (EHL) for patients were not evaluated, which may influence therapeutic outcome or trigger potential side effects. ${ }^{23,24}$

In summary, based on these two cases, we propose that the adrenocortical function of patients with severe thyrotoxicosis be evaluated around ${ }^{131} \mathrm{I}$ administration for. Determination of the basal serum cortisol level is an optional screening test in testing- limited institutions. For hemodynamically stable patients, high-dosage $(250 \mu \mathrm{g})$ ACTH stimulation test is advised. Adrenocortical insufficiency is conclusive when serum cortisol concentration cannot reach $18 \mu \mathrm{g} / \mathrm{dL}$ within $60 \mathrm{~min}^{28}$ Moreover, prophylactic glucocorticoid could be considered around the day of ${ }^{131} \mathrm{I}$ administration in critical patients with free thyroxine 2-3 times the upper limit of the normal. ${ }^{29}$ Methylprednisolone may be preferred to prednisolone to obtain a stable intra-therapeutic EHL of ${ }^{131} \mathrm{I} .{ }^{23}$ If suspected adrenal crisis does occur following RAI, we suggest performing random serum cortisol and plasma ACTH measurement immediately and giving intravenous hydrocortisone succinate and fluid resuscitation. The dose and course depend on individual varied conditions.

\section{Conclusion}

Although RAI therapy has been widely used and well tolerated for decades, ${ }^{3}$ it should be treated with caution for those with prolonged severe Graves' disease, as they may face an increased risk of relative adrenal insufficiency. Basal or stimulated cortisol might be evaluated around ${ }^{131} \mathrm{I}$ administration if available. Pretreatment with adequate glucocorticoid in necessity might be beneficial.

\section{Ethics and Consent Statement}

This study was performed in accordance with the principles of the Helsinki Declaration and approved by the Ethics Committee of Zhejiang University, School of Medicine affiliated Sir Run Run Shaw Hospital.

\section{Consent for Publication}

Written informed consents for publication were obtained from our patients.

\section{Funding}

This research did not receive any specific grant from any funding agency in the public, commercial or not-for-profit sector.

\section{Disclosure}

The authors declare that there are no conflicts of interest.

\section{References}

1. Teng W, Shan Z, Teng X, et al. Effect of iodine intake on thyroid diseases in China. $N$ Engl $J$ Med. 2006;354(26):2783-2793. doi:10.1056/NEJMoa054022

2. Ningyi J, et al. Guidelines for radioactive iodine therapy for graves' hyperthyroidism. Chin J Endocrinol Metabol. 2013;29(6):448-459.

3. Ross DS, Burch HB, Cooper DS, et al. 2016 American Thyroid Association guidelines for diagnosis and management of hyperthyroidism and other causes of thyrotoxicosis. Thyroid. 2016;26 (10):1343-1421. doi:10.1089/thy.2016.0229

4. Hoshiro M, Ohno Y, Masaki H, et al. Comprehensive study of urinary cortisol metabolites in hyperthyroid and hypothyroid patients. Clin Endocrinol (Oxf). 2006;64(1):37-45. doi:10.1111/j.1365-2265.20 05.02412. $\mathrm{x}$

5. Gallagher TF, Hellman L, Finkelstein J, et al. Hyperthyroidism and cortisol secretion in man. J Clin Endocrinol Metabol. 1972;34(6):919. doi:10.1210/jcem-34-6-919

6. Fredette ME, Topor LS. Graves' thyrotoxicosis leading to adrenal decompensation and hyperandrogenemia in a pediatric patient with salt-wasting congenital adrenal hyperplasia. Case Rep Endocrinol. 2018;2018:2359205.

7. Attaye I, van Andel M, Kooter AJ. More, less or both? BMJ Case Rep. 2018;2018. doi:10.1136/bcr-2017-222355

8. Skamagas M, Geer E. Autoimmune hyperthyroidism due to secondary adrenal insufficiency: resolution with glucocorticoids. Endocrine Pract. 2011;17(1):85-90. doi:10.4158/EP10069.CR 
9. Satoh T, Isozaki O, Suzuki A, et al. 2016 guidelines for the management of thyroid storm from The Japan Thyroid Association and Japan Endocrine Society (First edition). Endocr J. 2016;63(12):1025-1064. doi:10.1507/endocrj.EJ16-0336

10. Naik D, Jebasingh KF, Thomas N. Delayed diagnosis of Graves' thyrotoxicosis presenting as recurrent adrenal crisis in primary adrenal insufficiency. $J$ Clin Diagn Res. 2016;10(4):OD20-2. doi:10.7860/JCDR/2016/16395.7678

11. Bernet VJ. Thyroid hormone misuse and abuse. Endocrine. 2019;66 (1):79-86. doi:10.1007/s12020-019-02045-1

12. Karl M, Onumah BM, Cole J, et al. Hypocortisolemia in Graves hyperthyroidism. Endocrine Pract off J Am College Endocrinol Am Assoc Clin Endocrinol. 2009;15(3):220. doi:10.4158/EP.15.3.220

13. Agbaht K, Gullu S. Adrenocortical reserves in hyperthyroidism. Endocrine. 2014;45(1):136-143. doi:10.1007/s12020-013-9933-y

14. Price SA, Thondam S, Bondugulapati LNR, et al. Significant attenuation of stimulated cortisol in early Graves disease without adrenal autoimmunity. Endocrine Pract off $\mathrm{J} \mathrm{Am}$ College Endocrinol Am Assoc Clin Endocrinol. 2012;18(6):924. doi:10.4158/EP12002.OR

15. Johnson EO, Kamilaris TC, Calogero AE, et al. Experimentallyinduced hyperthyroidism is associated with activation of the rat hypothalamic-pituitary-adrenal axis. Eur J Endocrinol. 2005;153 (1):177-185. doi:10.1530/eje.1.01923

16. Mishra SK, Gupta N, Goswami R. Plasma adrenocorticotropin (ACTH) values and cortisol response to 250 and 1 microg $\mathrm{ACTH}$ stimulation in patients with hyperthyroidism before and after carbimazole therapy: case-control comparative study. J Clin Endocrinol Metab. 2007;92(5):1693-1696. doi:10.1210/jc.2006-2090

17. Tsatsoulis A, Johnson EO, Kalogera $\mathrm{CH}$, et al. The effect of thyrotoxicosis on adrenocortical reserve. Eur J Endocrinol. 2000;142 (3):231. doi:10.1530/eje.0.1420231

18. Satoh M, Aso K, Ikehara S, et al. A 3-year-old girl with Graves' disease with hypoglycemia following transient adrenal hyporesponsiveness. J Pediatr Endocrinol Metab. 2011;24(11-12):1047-1050. doi:10.1515/JPEM.2011.307

19. Fallahi P, Ferrari SM, Ragusa F, et al. Th1 chemokines in autoimmune endocrine disorders. J Clin Endocrinol Metab. 2020;105 (4):1046-1060. doi:10.1210/clinem/dgz289
20. Weetman A. Autoimmune thyroid disease. Endocrine. 2020;68 (2):258-260. doi:10.1007/s12020-020-02188-6

21. Kim M, Kim BH, Jang MH, et al. High neutrophil-to-lymphocyte ratio is associated with relapse in Graves' disease after antithyroid drug therapy. Endocrine. 2020;67(2):406-411. doi:10.1007/s12020019-02137-y

22. Mizokami T, Hamada K, Maruta T, et al. Painful radiation thyroiditis after ${ }^{131} \mathrm{I}$ therapy for graves' hyperthyroidism: clinical features and ultrasonographic findings in five cases. Eur Thyroid J. 2016;5 (3):201-206. doi:10.1159/000448398

23. Happel C, Kranert WT, Gröner D, et al. Focus on radioiodine-131 biokinetics: the influence of methylprednisolone on intratherapeutic effective half-life of $131 \mathrm{I}$ during radioiodine therapy of Graves' disease. Endocrine. 2021. doi:10.1007/s12020-020-02593-x

24. Halstenberg J, Kranert WT, Korkusuz H, et al. [Influence of glucocorticoid therapy on intratherapeutic biodistribution of $131 \mathrm{I}$ radioiodine therapy in Graves' disease]. Nuklearmedizin. 2018;57 (2):43-49. German. doi:10.3413/Nukmed-0941-17-11

25. Bonnema SJ, Hegedus L. Radioiodine therapy in benign thyroid diseases: effects, side effects, and factors affecting therapeutic outcome. Endocr Rev. 2012;33(6):920-980.

26. Vennard K, Gilbert MP. Thyroid storm and complete heart block after treatment with radioactive iodine. Case Rep Endocrinol. 2018;2018:8214169. doi:10.1155/2018/8214169

27. Nascif SO, Molica P, Correa-Silva SR, et al. Ghrelin and GHRP-6-induced ACTH and cortisol release in thyrotoxicosis. Pituitary. 2009;12(4):315-321. doi:10.1007/s11102-009-0181-3

28. Annane D, Pastores SM, Rochwerg B, et al. Guidelines for the diagnosis and management of critical illness-related corticosteroid insufficiency (CIRCI) in critically ill patients (Part I): society of Critical Care Medicine (SCCM) and European Society of Intensive Care Medicine (ESICM) 2017. Intensive Care Med. 2017;43 (12):1751-1763. doi:10.1007/s00134-017-4919-5

29. Bartalena L, Baldeschi L, Boboridis K, et al. The 2016 European Thyroid Association/European Group on Graves“ Orbitopathy Guidelines for the Management of Graves“ Orbitopathy. Eur Thyroid J. 2016;5(1):9-26. doi:10.1159/000443828
International Journal of General Medicine

\section{Publish your work in this journal}

The International Journal of General Medicine is an international, peer-reviewed open-access journal that focuses on general and internal medicine, pathogenesis, epidemiology, diagnosis, monitoring and treatment protocols. The journal is characterized by the rapid reporting of reviews, original research and clinical studies across all disease areas. The manuscript management system is completely online and includes a very quick and fair peer-review system, which is all easy to use. Visit http://www.dovepress.com/ testimonials.php to read real quotes from published authors. 